\title{
Standardization Of Clinical Documents THROUGH HL7 - FHIR FOR COLOMBIA
}

\author{
López Juan D. ${ }^{1}$ Moreno Luis. G. ${ }^{2}$ Anzola Diego ${ }^{3}$ López Francisco J. ${ }^{4}$ Salinas \\ Martha ${ }^{5}$ Vilardy Aldo ${ }^{6}$ García Stella ${ }^{7}$ and Lagos Arturo ${ }^{8}$ \\ ${ }^{1}$ Chief Executive Officer, Lv Consultores, Bogotá, Colombia \\ ${ }^{2}$ Executive Director, Lumon, Bogotá, Colombia \\ ${ }^{3}$ Software Architect, Lumon, Bogotá, Colombia \\ ${ }^{4}$ Technical Advisor, Lv Consultores, Tuluá, Colombia \\ ${ }^{5}$ Academic Advisor, Uniminuto, Bogotá, Colombia \\ ${ }^{6}$ Technical Advisor, Innova Ip, Bogotá, Colombia \\ ${ }^{7}$ Teaching researcher, ECCI University, Bogotá, Colombia \\ ${ }^{8}$ Teaching researcher, ECCI University, Bogotá, Colombia
}

\begin{abstract}
The importance of health sector in Colombia is notorously growing. In this article one of the major issues concerning the field is addressed, the interoperability of heath history. Due to the stablishment of standards , Colombia must begin a process to accomplish such an important necesity to offer an efficient and high quality health service. In this context, the standar FHIR is an example of successfull implementation of electronic health history in public sector. It's tought to be gradually implemented first in the beneficiary institution Rubén Cruz. Vélez Hospital and further in ther health institutions in the country.
\end{abstract}

\section{KEYWORDS}

Interoperability - HL7 FHIR - HCE - Archetypes

\section{INTRODUCTION}

The article shows the arising of a need that is evident in the electronic medical records of each hospital and clinic, because each one manages its own characteristics but there is no interoperability between state and private hospitals not even among those who share the same references. The case studied is funded by the Ministry of ICT and Colciencias and developed in the HOSPITAL RUBEN CRUZ VELEZ of Tulúa - VALLE. An electronic medical history focused on morbidity rate with 13 modules standardized with HL7 - FHIR is validated. In total 13 health centers and health posts are part of the study.

This platform will allow institutions with heterogeneous information systems to communicate with each other. One of the objectives of the work is to offer to the health community and especially the health Ministry as a guarantor of health in the country, the possibility of develop an interoperable route into the country through the HL7 standard - FHIR .

It is important to stress that as the need for semantic interoperability and exchange of EHR is presented, new standards and methodologies emerge to meet new needs and take advantage of new technologies. 
One of the standards developed under this need is Fast Healthcare Interoperability Resources ( FHIR ) [1 ] . FHIR Family is HL7 specification for the electronic exchange of health information. FHIR take advantage of the lessons learned from HL7 HL7 V2 and V3 to provide a specification for interoperability of health information in order to facilitate implementation.

HL7 - FHIR, is based on a set of basic modular components, describing the contents of clinical and administrative health historys that can be exchanged. That's the reason why this work is part of a solution through HL7 - FHIR standard, looking for demonstrating that the HL7 - FHIR standard is appropriate and effective for this innovation. Each HL7 feature developed based on standard is described.

\section{METHOD}

Each characteristic of different standards of electronic health history is explained, and the importance of working with HL7 - FHIR standard in the development of this work in hospital Ruben Cruz Velez de Tulua - Valle del Cauca is exposed.

\subsection{RELATED WORK}

At about the 90s research on the technical aspects of interoperability of EHR through different research projects started( Synapses [2 ], GEHR [3] ). One of the results of these projects has been transferred to standards backed by standardization bodies such as CEN (European Committee for Standardization), ISO and HL7 . The most striking result was the ISO standard EN 13606 on representation and communication of EHR [4 ] .

Maybe the main historical background of the beginning of HL7 in Colombia is the foundation of HL7 in Colombia, which began operating in March 2007. After research and interaction with other African countries involved in the operation the health sector, the use of the HL7 standard in Colombia for information, promotion and implementation was requested, highlighting HL7 as a standard for interoperability of health information at international level that integrates protocols, standards , encodings ( SNOMED , LOINC , DICOM, IHE , etc. ) and represents the benchmark in the industry on which leading provider of technology and ICT are making developments and technological advances to aspects of information exchange [5 ].

During 2010 and 2014, a study developed by the engineer Juan Diego Lopez in the technical aspects of interoperability of EHR through different research projects ( GS [6 ] , SFA [7 ] , AVC [8 ] started , ACM [9 ], LAM [10 ] ). One of the results of these projects was to participate in several international conferences and articles,. Besides, the IPS Climar in Bogotá is running a clinical history of cancer adapted to HL7v3 standard, thanks to these works. ] . .

\subsection{INTEROPERABILITY IN HEALTH SECTOR}

\subsubsection{ELECTRONIC HEALTH HISTORY}

Electronic medical records (EMR ) is the set of documents relating to the health of a patient registered electronically . Currently the HCE is considered a key to the efficient delivery of health services and, in particular, to ensure the quality of care and patient safety. Also, the clinical information stored in the EHR contributes to medical research and health professionals and public health training [11].

Research conducted over the past two decades has brought to light the clinical, ethical and technical requirements of EHR systems . These requirements have been consolidated by ISO 
technical specification that provides a benchmark for the development of the EHR [12 ] . In general, the most important challenges that information technology and communications face to successfully implement EHR are to provide confidence in information sharing and ensure that clinical information is interpreted correctly and safely. Following this purpose, health systems must interoperate at the semantic level, so that a system can understand the context and meaning of information provided by another.

\subsubsection{INTEROPERABILITY STANDARDS IN HEALTH SERVICE.}

In recent years, several studies funded by the European Commission have focused on interoperability of EHR in a broader sense. The RIDE [13 ] project defined a first roadmap in EHR IMPACT [14 ] analyzed the benefits of interoperable EHR systems and SemanticHEALTH [11 ] defined a roadmap for research and development of feasible solutions in the short and medium term to achieve interoperability of EHR .

The final report of SemanticHEALTH project defines several levels of interoperability between EHR systems. The most basic level is achieved when the communication is done HCE at document level, where consultations are limited to record dates and the provider of the information or document type. This level is called syntactic interoperability and is sufficient if the information requirement is that the documents must be legible for health professionals. However, the existence of several standards to represent the HCE hinders communication and exchange of information between health systems. The main standards organizations in Medical Informatics are working on harmonizing their standards. Although a single standard has emerged as a clear winner, Health Level 7 (HL7). Standards are a popular choice in many local communities in the United States, such as local integration in their networks and hospitals, as well as regional and national levels is an initiative of electronic health throughout the Americas. Several organizations play different roles in the development of interoperability standards in health care. There are standards organizations such as HL7 and DICOM.

\subsubsection{HL7}

HL7 (Health Level Seven) is a non -profit standards organization pertaining ANSI since 1987 which develops standards for the exchange of clinical and administrative data between health information systems . HL7 is the term is used to refer to both the organization and the set of messaging standards. Today, HL7 is an international community of health information experts who collaborate to develop standards for the exchange of information on health and interoperability of health systems.

HL7 produces both an electronic system for messaging standards and other standards such as standardized electronic structure and content to support interoperability systems . Messaging standards are available as HL7 HL7 v2 and v3 .

\subsubsection{HL7 v2}

HL7 version 2 is the standard in Medical Informatics more implemented in the world. However, despite its success, version 2 of the HL7 standard has certain interoperability problems mainly due to the flexibility to define messages that forces the participants in the communication entities to agree on the interpretation of messages [fifteen]. HL7 v2, was initially developed in 1989 in an ad hoc manner, it was based on integrating multiple systems together as hospital administrative and clinical systems. For example, hospitals are often independent in purchase and billing system respect to laboratory and they can not communicate automatically during installation. 
HL7 v2 is a standard that has been well adopted in hospitals and local communities, and with the support of most health care providers in information systems in North America . The ad hoc nature of HL7 v2 meant that it did not scale well in environments of multiple parts, ie information systems for jurisdictional environments ; for example, it lacks inherent HL7 v2 support global identifiers of the company. HL7 v2 also has a strong dependence of customization premises through the use of so-called "Z - segments " . System interfaces are designed to be $80 \%$ and are defined by the HL7 specifications and $20 \%$ for requirements particular by local application. A second drawback of HL7 v2 is the lack of a formal ontology in unifying concepts that are exchanged through various messages and interfaces [16] .

\subsubsection{HL7 v3}

HL7 Version 3 begins in the year 1995, overcoming the limitations of the previous version by proposing a formal methodology to define messages and the transition to XML syntax. All message specifications are defined from a generic information model known as RIM (Reference Information Model) [17 ]. This version of the standard also proposes a process for deriving the message models from RIM. Also, information models are designed to be associated with terminology systems and controlled vocabularies for some domains are defined. HL7 messages models v.3 are derived from RIM through a refining process to obtain a representation of implementable [ 18 ] messages.

For a particular domain, a D -MIM (Domain Message Information Model) model defined from entities RIM is obtained. Next, a R -MIM model (Refined Message Information Model ) containing the information necessary to define one or more HMD (Hierarchical Message Description) is constructed. HDM models are a tabular representation of a sequence of elements of an R -MIM independent of any technology implementation . Finally, the standard also defines the specification XML implementable Technology to express HMD in XML models. The documentation of the HDF development process is only 144 pages.

Finally, HL7 v3 aims to provide semantic interoperability, but ironically, because of the syntactical complexity in the field, it has been dramatic examples of misinterpretations leading to unaligned implementations and therefore a significant work network. An example of this is incompatible implementations Ontario and Alberta Provincial in United States where the patient records and dynamic behavior with respect to identity merge operations in spite of being developed using the same standard equipment [16 ].

\subsubsection{ISO EN 13606}

The technical committee CEN TC 251 is responsible for the development of standards in Medical Informatics in the European Union. The most notable result was the ISO standard EN 13606, which is the only complete EHR interoperability standard at international level. The objective of this standard is the definition of a rigorous and stable information architecture for communicating EHR, noted for the application of dual architecture model. In addition, the specification of the standard has been developed to preserve the clinical significance established by the author of the record and the confidentiality of the data as it is established by the author and patient. The ISO standard EN 13606 has five parts. The first two parts correspond to the two levels of dual architecture, reference model (part 1) and the specification for the exchange of archetypes (part 2). The latter defines the technical requirements to be fulfilled by the archetypes, as well as the syntax and semantics of the language for definition of archetypes (ADL). Part 3 includes guidance on the use of the reference model and design of archetypes and includes an initial vocabulary of terms that can be used to instantiate the reference model. In addition, Part 3 defines a set of archetypes that illustrate how to represent clinical information defined in other standards 
such as HL7. Parts 4 and 5 deal with security access to the HCE and specification of service interfaces and messages for communication EHR. Today the tools available for this standard are slim. Among them, the platform that has contributed to the dissemination and implementation of the standard is LinkEHR [19], which consists of an editor of archetypes (LinkEHR-Ed), a standardization tool of clinical data in legacy systems (LinkEHR Studio) and a web viewer HCE (LinkEHR Viewer), among other tools. Recently, it has emerged the association IN 13606 [20] for the purpose of promoting and spreading the standard.

\subsection{FHIR HL7 StANDARD. FAST HEALTHCARE INTEROPERABILITY RESOURCES}

For background on implementations HL7 v3, including ineligibility for the United States, HiTech financed in January 2011, in conjunction with the Board of Governors, the beginning of a fresh look to the HL7 standard for this settle group work, with the aim of examining how HL7 messaging standard could be improved. This inspired the organization with an independent group of architects HL7 to begin a discussion to give the new approach for the exchange of health information where initially "Health Resources - RFH" would be called, and was later renamed to "Quick Resources for interoperability in Health - FHIR "[21]. This new approach was based on REST principles described by Fielding [22]. FHIR effort aims to simplify and accelerate HL7 adoption to an easily consumable, but robust way, and the use of open Internet standards whenever possible.

Easily consumable format for standard avoids the need for customized and complex tools. The standard contains application examples for all devices and reference implementations of various platforms, including live test servers available over the Internet. The authors claim that HL7 v3 was a good base, and has lead to FHIR through v3 learning.

\subsubsection{STANDARD FHIR DEVELOPMENT Process}

While messaging HL7 v2 followed a process of ad hoc development and HL7 v3 had followed a process led by the top -down model-driven process rigidly defined by the HDF, FHIR uses an incremental and iterative approach to the development of the standard . The incremental development refers to the development of small pieces at a time. Additional parts are applied and tested in learning that is fed back into the design process very quickly. After that, it continues to the iterative part of the process [16].

\subsubsection{FHIR ARTIFACTS}

FHIR aims to define the key entities involved in health care, such as Resource Information Exchange. Each resource is a clear specification to describe the FHIR [16].

The following are the Resources and Attributes:

- Resources should have a clear boundary, which matches one or more logical in the fields of the transaction.

- Resources should differ from each other in meaning, not simply use (eg different ways to use a lab report should not lead to different resources).

- Resources need to have a natural identity.

- Resources must be very common and used in many different business transactions.

- Resources should not be specific or sufficient to exclude support for a wide range of commercial transactions details.

- Resources should be mutually exclusive. 
- Resources should use other resources, but should be more than just compositions of other resources; each resource must introduce a new content.

- Resources should be organized in a logical framework based on the commonality of resources and what it unites.

- Resources should be large enough to establish context; resources that contain only a few attributes are probably too small to provide significant business value.

\subsubsection{WHY FHIR ?}

Resources are normally : Patient, devices and documents. At the time of this writing there are 32 defined with many more resources into consideration. The development team estimates there will be about 150 resources defined in total. FHIR process designers have stated that "FHIR tries to keep the design rigor representing resources $\mathrm{v} 3$ in a single transport ". In common structures of reusable classes, HL7 v3 is defined as types elements of common messages. the FHIR is important and relevant by:

The number of CMETs in the 2010 Edition Regulations HL7 v3 specification was 194, while the number of resources defined in FHIR for February 2013 ( after about 18 months of development ) is about 32. This represents a drastic reduction in the number of concepts which the application requires [ 16]

\section{Solution of the Problem}

Within the solution, the whole process of architecture, specifications, implementation, chosen standard and technical specification message and its structure are stablished. It's important to note that the development methodology used is Scrum, the requirements units are called Product Backlog Item (GDP) and the tool we use is Team Foundation Server.

\subsection{ARCHITECTURE DiagRAM}

The HL7 system is designed as follows:

The system consists of three layers where each has a specific function, besides it allows better control on the operation of the application. Functions of the layers are:

- Presentation Layer: In this layer the application forms are found. It was developed with the MVC pattern and designed with the bootstrap INSIPINA template.

- Application Layer: The application layer function will be the connection between the presentation layer and the data layer where we have 3 layers within for better fluidity in data processing.

- Data Access: it allows to create the connection to the database with the Entity Framework technology and also helps in mapping tables which are in the database.

- Business tasks: it has the function to receive data from the api web services valid to have a successful registration.

- Rest Services: REST services are charged on a Web Api library of Visual Studio. 


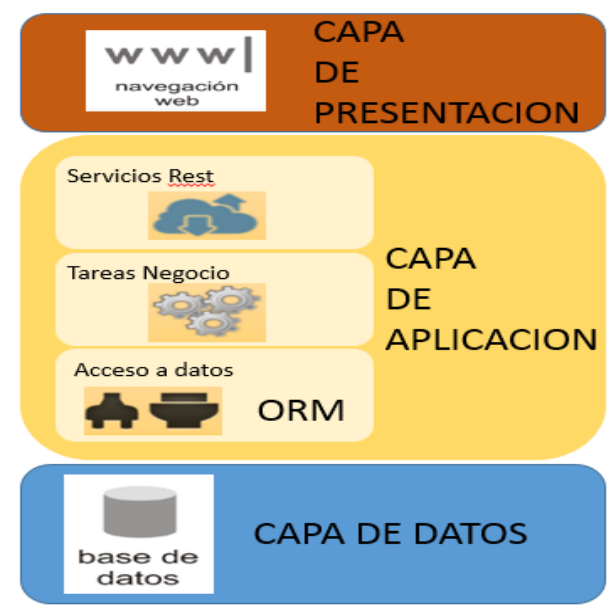

Figure 1. Layer Data : The data layer is made in Microsoft SQL Server. Source: The authors

\subsection{SPECIFICATION DEPLOYMENT}

The following diagram depicts the architecture execution developed, however the design allows a single server with DB server role and Server Software be necessary for execution

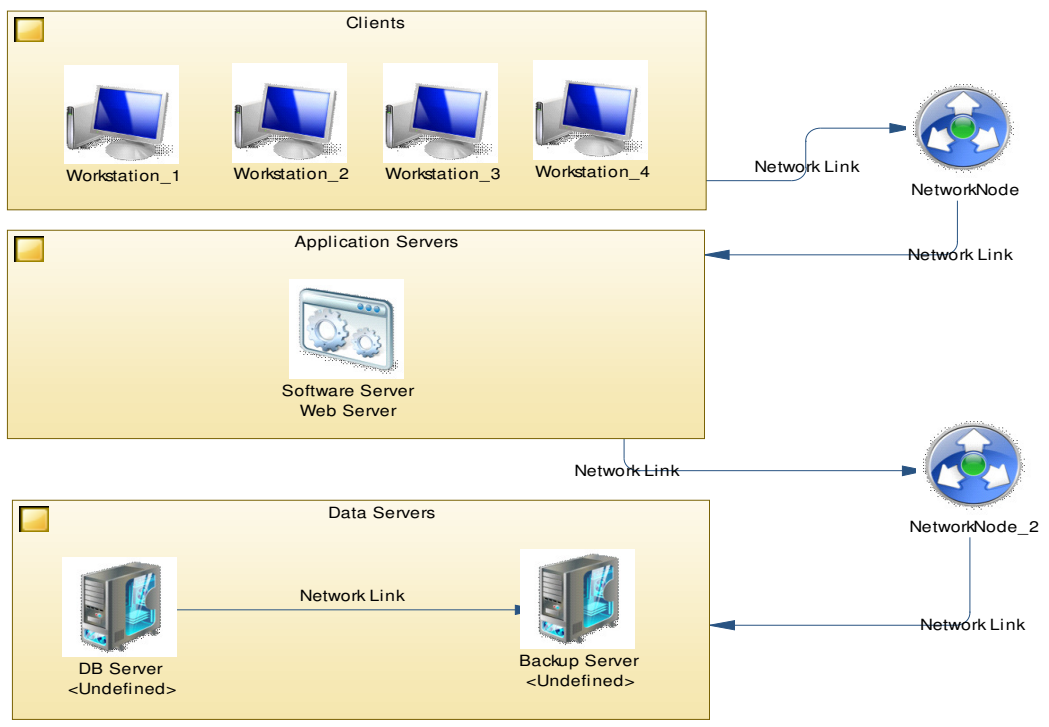

Figure 2. Execution Architecture Developed Source: the authors

\subsection{IMPLEMENTATION DETAILS}

The graphic design was developed following the guidelines of the presented image . The solution was designed under Visual Studio 2012 . The communication protocol between clients and the application is HTTP. 
International Journal of Computer Science \& Information Technology (IJCSIT) Vol 8, No 6, December 2016

\subsubsection{ASSUMPTIONS AND DEPENDENCIES.}

Customers have installed versions of browsers that allow and support the execution of javascript.

\subsubsection{EXTERNAL INTERFACES REQUIREMENTS}

User Interfaces . User interfaces must be designed according to the guidelines established .

\subsubsection{HARDWARE INTERFACES}

- Customers

Hardware: Processor 32-bit or 64 - bit 1 GHz or more . 1GB RAM ( 32 - bit) 2GB RAM ( 64 bit) . 16GB of available hard disk space ( 32 - bit) or 20 GB ( 64- bit) . Peripherals : Keyboard and mouse.

- Application Server

Hardware: 1Ghz Processor ( 32 - bit) or $1.4 \mathrm{Ghz}$ ( 64 - bit) . 2GB RAM ( 32 - bit) 4GB RAM ( 64 - bit) or more . 20GB hard disk or more.

- DBServer Hardware: 1Ghz Processor ( 32 - bit) or 1.4 Ghz ( 64 - bit) . 2GB RAM ( 32 - bit) 4GB RAM ( 64 - bit) or more 20GB hard disk or more.

- Backup Server.

Hardware: 1Ghz Processor ( 32 - bit) or $1.4 \mathrm{Ghz}$ ( 64 - bit) . 2GB RAM ( 32 - bit) 4GB RAM ( 64 - bit) or more . 20GB hard disk or more.

\subsubsection{SOFTWARE INTERFACES}

- Customers

Software: Windows 7+ or Preferably operating systems able to running any of the indicated browsers and that have support for updates.

Browser : Firefox (Current -1) or Current, Chrome (Current -1) or Current, Opera $>=12.1 \mathrm{x}$, Safari 5.1+, IE 9+

- Software Server:

Software:

- Microsoft Windows Server 2008 R2 Enterprise.

\subsubsection{ADDITIONALS}

- Development Tool : Microsoft Visual Studio 2012

- Communication interfaces. For communication between clients and software HTTP was used . . To connect the application server with the data server TCP / IP was used. 


\subsection{FHIR Our standard}

FHIR offers many improvements over existing standards:

- A strong focus on application - fast and easy to implement (several developers have simple interfaces that work in one day).

- Multiple execution libraries, many examples available to start development.

- The specification is free for unrestricted use

- Interoperability out-of-the-box- resource base can be used as ti is, but can also be adapted to local requirements.

- Path of evolutionary development of HL7 version 2 and CDA - standards can coexist and benefit each other.

- Solid Web -based standards XML, JSON, HTTP, OAuth, etc.

- Support for RESTful architectures and continuous exchange of information also through messages or documents.

- Concise and easy to understand specifications

- A human readable format wire for easy use by developers.

- The analysis based on solid ontologies with a rigorous and formal assignment of correction.

\subsubsection{FLEXIBILITY}

A central challenge for health standards is how to handle the variability caused by various care processes. Over time, more and optionality fields are added to the specification, adding little cost and complexity of the resulting implementations. The alternative is based on custom extensions, but they also create many problems of application.

FHIR solves this problem by defining a simple framework to extend and adapt existing resources. All systems, regardless of how they develop, can easily read these extensions and definitions of extension can be recovered using the same framework as the recovery of other resources.

In addition, each resource leads to a representation of human readable text using html as a display option for clinical safety reserve. This is particularly important for complex clinical information in which many of the systems take a simple document/ textual -based approach.

\subsubsection{FHIR IMPLEMENTATION PROCESS}

FHIR is published as a draft standard for trial use . During the use test stage, HL7 actively monitors implementations in order to further improve the specification, and is able to respond to their needs . Because of the many advantages FHIR offers, the use test is already starting at this time. FHIR implementation is carried out through the following processes:

- Capturing patient data, consultation and procedures through Electronic Medical Record System

Information of electronic medical records is exposed through REST services with the standardization notation defined for FHIR . An example of the response to the resource query is shown Patient : 


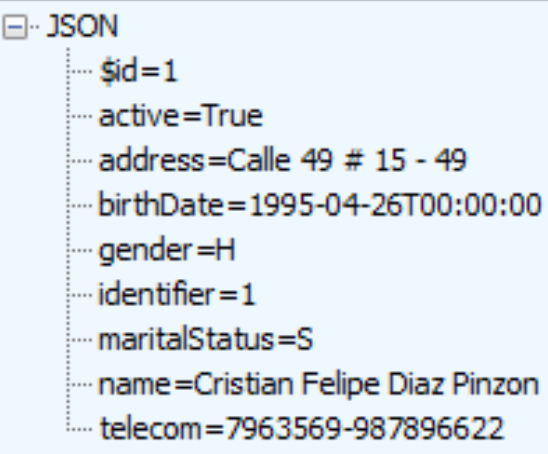

Figure 3. Example of Resources : PATIENT Source: the authors

- The customer or the receiving entity must have an information system that can consume REST services in accordance with the notation defined and standardized by FHIR to retrieve data from the clinical history.

\section{Conclusions}

One of the objectives of this paper is to present the reason of the decision to work with the FHIR standard. In order to achieve this objective, the evolution of the different standards such as HL7 v2 and v3 and ISO 13606 for the interoperability of health systems, was studied. On this basis each standard were studied and analyzed and a comparison was made.

The FHIR standard has attracted a lot of attention from all relevant community because of its simplicity (following the REST architecture).

There are still ambiguities about how the FHIR HL7 standard should use the strengths of its predecessors (HL7 V2 and V3 ). However, it is clear that the authors of FHIR take advantage of the extensive experience gained in the implementation of their ancestors, which will dramatically improve the state of information communication between health systems .

In conclusion, the implementation of standards HL7 Electronic health history was developed using HL7 FHIR technology which corresponds to the latest efforts of the HL7 organization in implementing interoperable services .

FHIR has fast interoperability of health resources, it is a framework of standards created by HL7 next generation. FHIR combines the best features of HL7 v2, v3 and CDA product lines, and take advantage of the latest web standards by applying a strict focus on applicability.

FHIR offers many improvements over existing standards:

- A quick and easy approach to implement application.

- Multiple execution Libraries and many available examples to start development.

- The specification is free for unrestricted use.

- Evolutionary development of HL7 version 2 and CDA - standards that can coexist and benefit each other. 
- Solid Web standards based on XML, JSON, HTTP, OAuth, etc.

- Support for RESTful architectures and continuous exchange of information through messages or documents.

- Concise and easy to understand specifications

- A readable format for easy use by developers.

Finally, the development implemented in the Hospital Ruben Cruz Velez, with FHIR, is one of the first developments in Latin America for standardized clinical history. A success from every point of view particularly in the human capital in their organizational learning as institution, in interpreting the data was observed. I is also highlighted the important rapprochement with the government of Valle del Cauca and the Mayor of Cali, who showed interest in implementing tan interoperable electronic health historys throughout the Department.

As recommendation it's important to ensure that these projects follow a second phase to achieve interoperability within all the hospitals and clinics into the municipality of study. The Ministry of Health should visualize the future that an EHR standard can give for the quality of health service.

\section{ACKNOWLEDGEMENTS}

Mainly Colciencias Doctor Emiro Tovar and Ministry of ICT Doctor Nestor Fajardo , with its valuable contribution to the research project to fruition towards the Latin American Research, a special thanks to the Mayor of the City of Tulua , the Lord Mayor Gustavo Velez and Secretary TIC Alexander Andrade, believing the project to be linked in the city of Tulua, and finally thank the staff of the Hospital Ruben Cruz Vélez, Doctor Devia, Montano, Jose a and especially to Dr. Julio Cesar Toro, for giving us the doors Ruben Cruz Hospital open Velez beneficiary Project funded research by Francisco Jose de Caldas COLCIENCIAS Fund, Ministry of information Technology and communications Mintic, ECCI University, Innova \& Ip LTDA - Project No. $535-2015$

\section{REFERENCES}

[1] Fast Healthcare Interoperability Resources (FHIR) Specification n.d. Avalable http://hl7.org/implement/standards/fhir/ (last access, February, 2016).

[2] Grimson, W., Berry, D., Grimson, J., Stephens, G., Felton, E., Given, P. y O’Moore, R. Federated healthcare record server-the synapses paradigm. International Journal of Medical Informatics, vol. 52(1), pages 3-27, 1998.

[3] Ingram, D. The good european health history. Health in the new communication age, MF Laires, MF Ladeira and JP Christensen (Eds), IOS, pages 66-74, 1995.

[4] ISO. Health informatics - electronic health history communicatio standard (ISO/EN 13606). Available http://www.iso.org/ iso/. (last access, April, 2016).

[5] B. Blazona and M. Koncar, "HL7 and DICOM based integration of radiology departments with healthcare enterprise information systems," Int. J. Med. Inf., vol. 76, Supplement 3, pp. S425-S432, Dec. 2007.

[6] Karen Madelaine Gordo González Marnel Antonio Sánchez Moreno. Historia clínica general utilizando el estandar HL7 por mensaje de texto. 2011. Available in http://www.umb.edu.co/biblioteca/

[7] Diana Carolina Salazar Andrés Leonardo Forero; Nestor Acevedo Romero. Sistema de Comunicación de historias Clínicas bajo standards (ISO13606- HL7 CDA) Que permitan la Interoperabilidad Entre los Sistemas de Información de Salud. 2011. Available in. http://www.umb.edu.co/biblioteca/

[8] Diana Marcela Avendaño Alarcón Leidy Viviana Verdugo Santana; Cortés Machuca, Jonathan. Desarrollo de una historia clínica de Cáncer aplicando el estándar HL7 para Climar. 2013. Available en http://www.umb.edu.co/biblioteca/ 
[9] ACM [4], Diego Alejandro Ariza Medina Santiago Camargo Marin; Luis Alejandro Medina Delgado. Parametrización de la historia clínica y tipos de enfermedades según HL7 versión 3. Para un caso colombiano. 2013. Available en http://www.umb.edu.co/biblioteca/

[10] Heidy Viviana. Leal Vanegas Alarcón Ballén, Danny Fabián, Medina Castro, Néstor Fernando. Sistemas de Información para la Gestión de Etiquetas de Medicamentos utilizando el Estándar de HL7. 2014. Available en http://www.umb.edu.co/biblioteca/

[11] Stroetmann, V. N., Kalra, D., Lewalle, P., Rector, A., Rodrigues, J. M., Stroetmann, K. A., Surjan, G., Ustun, B., Virtanen, M. y Zanstra, P. E. Semantic Interoperability for Better Health and Safer Healthcare. Deployment and Research Roadmap for Europe. Available en ISBN-13:978-92-7911139-6.

[12] ISO 18308 Health informatics - Requirements for an electronic health history architecture. 2011. Available en http://www.iso.org/iso/home/store/catalogue_tc/catalogue_detail.htm?csnumber=52823 (last access, April, 2016).

[13] Dogac, A., Namli, T., Okcan, A., Laleci, G., Kabak, Y. y Eichelberg, M. Key issues of technical interoperability solutions in ehealth and the ride project. Software R\&D Center, Dept. of Computer Eng., Middle East Technical University, Ankara, vol. 6531, 2007.

[14] Dobrev, A., Jones, T., Stroetmann, V., Stroetmann, K., Vatter, Y. y Peng, K. Interoperable ehealth is worth it-securing benefits from electronic health historys and eprescribing. Bonn/Brussels: European Commission on Information Safety and Media, 2010.

[15] Eichelberg, M., Aden, T., Riesmeier, J., Dogac, A. y Laleci, G. B. A survey and analysis of electronic healthcare record standards. ACM Comput. Surv., vol. 37(4), pages 277-315, 2005. ISSN 0360-0300.

[16] Duane Bender, Kamran Sartipi HL7 FHIR: An Agile and RESTful Approach to Healthcare Information Exchange. IEEE 26th International Symposium on Computer-Based Medical Systems (CBMS). Pages 326 -33.June 2013.

[17] Health Level Seven. HL7 Reference Information Model. 2013. Available en http://www.hl7.org/implement/standards/rim.cfm (last access, April, 2016).

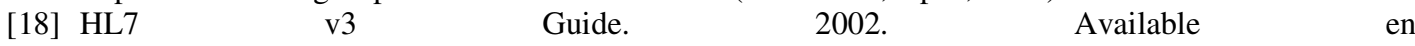
http://www.hl7.org/documentcenter/public/wg/mnm/hdf/v3guide.doc (last access, April, 2016).

[19] IBIME Group. LinkEHR Normalization Platform. 2013. Available en http://www.linkehr.com/ (last access, April, 2016).

[20] ISO EN 13606 Association. 2013. Available en http://www.en13606.eu (last access, April, 2016).

[21] FHIR: Fast healthcare interoperability resources.http://hl7.org/implement/standards/fhir/ (last access, April, 2016).

[22] R. T. Fielding. Architectural Styles and the Design of Network-based Software Architectures. PhD thesis, University of California, Irvine, 2000.

\section{Authors}

Engineer Juan Diego Lopez Vargas.

Telecommunications Engineer, Specialist Magister and doctoral student in Integration of IT in Organizations of the Polytechnic University of Valencia Spain. He is currently CEO and director of the Company LV Consultants SAS, was to held in administrative office and teaching in different institutions of higher education in Colombia, which takes about 10 years of experience in the structuring and execution of $\mathrm{R}+\mathrm{D}+\mathrm{i}$.

Luis Gabriel Moreno Engineer, Systems Engineer, MBA, and Ph.D. candidate in Computer Science. Currently legal representative of the company Lumon S.A.S, takes about 8 years experience in the IT sector in Colombia.

Anzola Diego engineer, Systems Engineer and Master in Computer Science, Expert in software architecture, with more than 8 years of experience in the IT sector.

Francisco Javier Lopez Engineer, Systems Engineer, a specialist in software engineering with over 20 years experience in the IT sector.

Lic. Martha Salina. License in Education, Candidate Doctor of Education, more than 20 years of experience in education. Currently Vice Chancellor Academic Uniminuto headquarters of Cundinamarca. 
International Journal of Computer Science \& Information Technology (IJCSIT) Vol 8, No 6, December 2016

Aldo Vilardy Engineer, Systems Engineer, you project structuring expert with experience of 5 years in sectPr IT.

Engineer Arturo Lagos, Dr. Engineer Computer Systems, with 20 years of experience in the ICT sector

Stella Garcia Engineer, Systems Engineer, Master of Sciences Computations with over 20 years experience in the IT sector. 